\title{
Gait Recognition Method by Combining Legs Region with Entire Gait Image
}

\author{
Li Kai \\ College of computer science and technology, Hebei \\ University \\ Baoding, China \\ Email: likai@hbu.edu.cn
}

\author{
Wang Guochao \\ College of computer science and technology, Hebei \\ University \\ Baoding, China \\ Email: lvyemimeng@sina.com
}

\begin{abstract}
Gait recognition method based on the feature combination of gait image and its region bounded by legs is proposed. Firstly, all the Eigenvectors correspond to nonnegative Eigenvalues of the entire scatter matrix of training samples are selected to compose a lower-dimension transform space. In this transform space, in order to overcome the small-sample-size problem, a regularization term is added to each sample class covariance matrix. And a new criterion function is established. By computing this optimization problem, and the eigen-matrix is made up by some eigen-vectors. Based on this method, the features of gait image and its region bounded by legs are combined to represent gait features. Finally, three gait databases of Chinese Academy of Sciences, CASIA A, CASIA B and CASIA $C$, are selected and the minimum distance classifier are used to verify the effectiveness of presented method.
\end{abstract}

Keywords-Gait Recognition; Gait Energy Image (GEI); Region Bounded by Legs (RBL); Feature combination; Regularization term

\section{INTRODUCTION}

Gait recognition is a new biometric recognition technology, it is mainly to identify a person by the pattern or style of walking. Compared with other biometric technologies, such as face recognition, gait recognition can identify a person at a distance, and need not to touch them. Gait recognition is a most promising biometric technology at a distance. At present, existing methods in gait recognition can be divided into model-based and model-free methods ${ }^{[1]}$. Model-based tend to recover the underlying mathematical construction of gait with a structure motion model, and the information of changes of joints of torso are collected to identify pedestrians when they are walking. However, the performance of the methods suffers from poor localization of the torso and difficult extraction of underlying models from gait sequences ${ }^{[2]}$. The other method is model-free, and it also can be classified into two categories. One kind of model-free method preserves temporal information in recognition and training states, and then identifies the human. Hence, the disadvantage for the method is the high computational complexity of sequence matching during recognition and the high storage requirement of the dataset. Another kind of model-free method converts a sequence of images into a single gait energy images, and then based on gait energy images, the temporal information in gait sequences are extracted and continue to identify pedestrian. This method is effective in reducing the number of training samples, thereby reducing the computational complexity. However, the performance of this type of methods is intensely affected by the background noise or covariate factors ${ }^{[3]}$ such as changes of the subject apparel and load carrying conditions. On the other hand, since gait data is frequently interspersed with the background noise or covariate factors, the classification could be misguided.

In order to study the gait recognition deeply, researchers have done a lot of works on it. Han et al. ${ }^{[4]}$ proposed a gait energy image (GEI) to construct some real and synthetic gait templates, and a good result was obtained on USF gait dataset. Roy et al. [5] introduced Pose Energy Image and Pose kinematics features which results shape and dynamics information. PCA and LDA techniques are applied for dimensionality reduction and to extract discriminative features. CMU's Mobo and USF dataset have been used by the authors for experiment and obtained acceptable results. Guo et al. ${ }^{[6]}$ proposed a method which based on mutual information to select a gait feature subset, by selecting some features which the value of mutual information are largest, and these features are composed a subset. Then the features of the subset are classified by SVM, and it and obtained a better result in Southampton HiD gait database. Mohan et al. ${ }^{[7]}$ proposed a method of gait recognition based on Local Binary Pattern (LBP). The Local Binary Pattern (LBP) is applied to Gait energy image and to the region bounded by legs (RBL) of Gait Energy Image. Extracted LBP values from GEI and RBL in GEI is represented in terms of interval valued type symbolic features. And then the Similarity between train samples and test samples are used to classify. The results on CASIA B, a kind of gait dataset of Chinese Academy of Sciences, are good but because of LBP only considered the relationship between the central pixel and neighboring pixels, and the relationship of contrast between them is ignored, so it did not obtained the good results on CASIA C.

Gait of individual may vary because of change in clothes, carrying a bag, wearing a different shoe types and in some particular time, some unusual way of walking or some special condition of walking may affect the recognition results ${ }^{[8]}$, so a new method is proposed as follows.

The remainder of this paper is organized as follows: Section II describes the details of the proposed method. Experimental results and analysis are provided in Section III, and Section IV concludes the paper. 


\section{GAIT RECOGNITION METHOD}

Gait recognition method mainly used to extract the features of both the gait images of pedestrian and the images of region bounded by legs(RBL), the dimension was reduced by PCA and then RLDA ${ }^{[9]}$ is used to extract features of the samples, at last the minimum distance identification method is used to identify the human.

\section{A. Gait Energy Image(GEI)}

In order to reduce the complexity in characterizing a gait, the silhouettes of a gait sequence are aggregated using the idea proposed in [1] to produce a single image called Gait Energy Image (GEI). GEI for a gait sequence is computed by taking the average of all silhouettes over a gait sequence and is defined as follows:

$$
\mathrm{GEI}=\mathrm{G}(\mathrm{x}, \mathrm{y})=\frac{1}{T} \sum_{t=1}^{T} I(x, y, t),
$$

where $\mathrm{T}$ is the number of frames in a gait sequence, $\mathrm{I}$ is a gait silhouette image, $\mathrm{x}$ and $\mathrm{y}$ are pixel coordinates and $\mathrm{t}$ is the frame number in a sequence of silhouettes in a gait sequence. Generally, before achieving the gait energy images, the gait images can be normalized to the same height and width and aligned along the upper part of the center of mass in the horizontal direction.

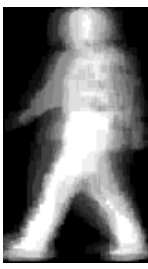

(a)



(b)

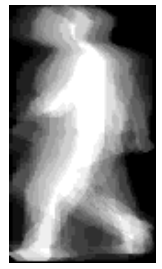

(c)

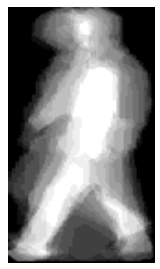

(d)
Fig.1 The GEI of pedestrians in four different conditions

Fig. 1 shows few examples of GEIs computed for same subject in three different instances (a) normal, (b) carrying a bag, (c) with coat and (d) shoot by infrared camera.

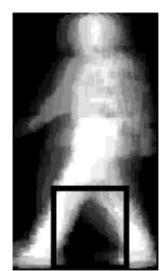

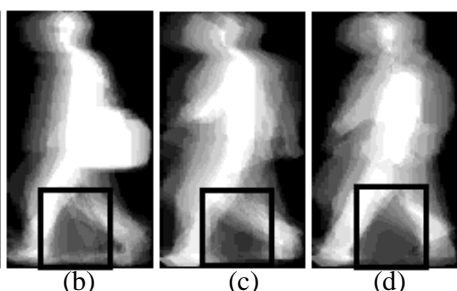

Fig. 2 The RBL of GEI
Fig. 2 shows the same GEIs with RBL area marked in the square box (a) normal, (b) carrying a bag, (c) with coat and (d) shoot by infrared camera.

\section{B. Feature Extraction}

Suppose gait energy images sets $G=\left\{G_{i}^{j} \mid G_{i}^{j} \in R^{n}\right\}$ is a set which includes $\mathrm{N}$ samples which belong to $\mathrm{C}$ categories, the samples of class $i$ can be represent as $G_{i}=\left\{G_{i}^{j} \in R^{n}, j=1,2, \ldots, N_{i}\right\}, \mathrm{N}_{\mathrm{i}}$ is the number of the samples of class $i$ and $\sum_{i=1}^{C} N_{i}=N$. Each $G_{i}^{j}$ is a vector, it represents a gait energy image, and $\mathrm{G}$ includes $\mathrm{N}$ samples of gait energy images. These samples belongs to one of $\mathrm{C}$ pattern classes $\left\{\mathrm{G}_{1}, \mathrm{G}_{2}, \ldots, \mathrm{G}_{\mathrm{C}}\right\}$, where $\mathrm{C}<\mathrm{N}$. $\mathrm{S}_{\mathrm{b}}, \mathrm{S}_{\mathrm{w}}$ and $\mathrm{S}_{\mathrm{t}}$ denote the betweenclass scatter matrix, within-class scatter matrix and total scatter matrix, respectively. Because of $G_{i}^{j}$ is a high dimension vector, and $\mathrm{S}_{\mathrm{t}}$ often is a singular matrix. So it is hard to extract features directly. In order to solve this problem, PCA is used to reduce the dimension of the vectors before feature extraction.

Suppose $\left\{\beta_{1}, \beta_{2}, \ldots, \beta_{n}\right\}$ are $\mathrm{n}$ orthonormal eigenvectors of $\mathrm{S}_{\mathrm{t}}$, and the first $\mathrm{m}\left(\mathrm{m}=\right.$ rank $\left.\mathrm{S}_{\mathrm{t}}\right)$ ones are corresponding to positive eigenvalues. Define the subspace $\Phi_{t}=\operatorname{span}\left\{\beta_{1}, \beta_{2}, \ldots, \beta_{m}\right\}$ and its orthogonal complement can be denoted by $\Phi_{t}^{\perp}=\operatorname{span}\left\{\beta_{m+1}, \beta_{m+2}, \ldots, \beta_{n}\right\}$. We can know from [10], for any arbitrary $\varphi \in R^{n}, \varphi$ can be denoted by $\varphi=\mathrm{X}+\xi$, where $\mathrm{X} \in \Phi_{\mathrm{t}}$ and $\xi \in \Phi_{\mathrm{t}}^{\perp}$. And it must satisfy $\mathrm{J}(\varphi)=\mathrm{J}(\mathrm{X})$. That is to say, we can conclude that all optimal discriminant vectors can be derived from $\Phi_{\mathrm{t}}$ without any loss of the optimal discriminatory information with respect to Fisher criterion $\mathrm{J}(\mathrm{X})$. That is to say, $G_{i}^{j}$ is reduced to m-dimensional Euclidean space without any loss of the optimal discriminatory information by PCA, and a PCA transformed space $\mathrm{R}^{\mathrm{m}}$ is obtained.

In order to facilitate representation, the between-class scatter matrix can be expressed as follows:

$$
\mathrm{S}_{\mathrm{b}}=\mathrm{HH}^{\mathrm{T}} \text {, }
$$

where

$$
\mathrm{H}=[\mathrm{H} 1, \mathrm{H} 2, \ldots, \mathrm{Hc}] . \quad \bar{G}_{i}=\left(1 / N_{i}\right) \sum_{j=1}^{N_{i}} G_{i}^{j}
$$$$
H_{i}=\left(N_{i} / N\right)^{1 / 2}\left(\bar{G}_{i}-\bar{G}\right) \text {, and } \bar{G}=(1 / N) \sum_{i=1}^{c} \sum_{j=1}^{N_{i}} G_{i}^{j} \text {. }
$$

Now, the problem is how to find the optimal discriminant vectors in $\Phi_{t}$. By linear algebra theory, $\Phi_{t}$ is isomorphic to $\mathrm{m}$ dimensional Euclidean space $\mathrm{R}^{\mathrm{m}}$. And the corresponding isomorphic mapping is $\mathrm{X}=\mathrm{PY}$, where $\mathrm{P}=\left(\beta_{1}, \beta_{2}, \cdots, \beta_{\mathrm{m}}\right)$, $\mathrm{X}$ $\in \Phi_{\mathrm{t}}, \quad \mathrm{Y} \in \mathrm{R}^{\mathrm{m}}$, By the isomorphic mapping $\mathrm{X}=\mathrm{PY}$, the criterion function $\mathrm{J}(\mathrm{X})$ becomes

$$
\begin{aligned}
& \qquad \tilde{J}(Y)=J(X) \\
& =\frac{Y^{T}\left(P^{T} S_{b} P\right) Y}{Y^{T}\left[\eta\left(P^{T} S_{b} P\right)\right] Y+Y^{T}\left(P^{T} S_{w} P\right) Y}, \\
& =\frac{Y^{T} \tilde{S}_{b} Y}{\eta\left(Y^{T} \tilde{S}_{b} Y\right)+Y^{T} \tilde{S}_{w} Y} \\
& \text { where } \quad \tilde{S}_{b}=P^{T} S_{b} P=P^{T} H H^{T} P=\left(P^{T} H\right)\left(H^{T} P\right)=\tilde{H} \tilde{H}^{T}
\end{aligned}
$$

$\tilde{S}_{w}=P^{T} S_{w} P$, and that means $\tilde{J}(Y)$ can act as a new criterion function like Fisher criterion function. If we get the optimal discriminant vectors based on $\tilde{J}(Y)$. That is to say, the optimal discriminant vectors are also obtained based on $\mathrm{J}(\mathrm{X})$. 
So $\tilde{J}(Y)$ act as Fisher criterion function, The optimal discriminant vectors based on $\mathrm{J}(\mathrm{X})$ are also obtained by solving the optimal discriminant vectors of $\tilde{J}(Y)$.

In PCA transformed space $\mathrm{R}^{\mathrm{m}}$, work out the $\tilde{H}^{T} \tilde{H}$ 's orthonormal eigenvectors $\left(\alpha_{1}, \alpha_{2}, \ldots, \alpha_{C}\right)$. Suppose the first 1 ones are corresponding to positive eigenvalues, Let $E_{l}=\left[\alpha_{1}, \ldots, \alpha_{l}\right], l \leq C-1$. Calculate the first 1 most significant eigenvectors $\left(U_{l}\right)$ of $\tilde{S}_{b}$ and their corresponding eigenvalues ( $\Lambda_{l}$ ) by $U_{l}=\tilde{H} E_{l}$ and $\Lambda_{l}=U_{l}^{T} \tilde{S}_{b} U_{l}$. Let $\mathrm{K}=U_{l} \Lambda_{l}^{-1 / 2}$. Work out eigenvalues $\left(\Lambda_{w}\right)$ and eigenvectors $(\mathrm{Z})$ of $\mathrm{K}^{T} \tilde{S}_{w} \mathrm{~K}$, $Z=\left[Z_{1}, \ldots, Z_{l}\right]$ sorted in increasing eigenvalue order. Choose the first $\mathrm{d}(\mathrm{d} \leq \mathrm{l})$ eigenvectors in $\mathrm{Z}$, let $Z_{d}=\left[Z_{1}, \ldots, Z_{d}\right]$ and $\Lambda_{d}$ be the chosen eigenvectors and their corresponding eigenvalues, respectively. And return $V=\left(Y_{1}, \ldots, Y_{d}\right)=K Z_{d}\left(\eta I+\Lambda_{d}\right)^{-1 / 2}, \mathrm{~V}$ is the eigen matrix based on $\tilde{J}(Y)$, and the optimal discriminant vectors based on $\mathrm{J}(\mathrm{X})$ are also obtained. Then the linear discriminant transformation can be defined as follows:

$$
\Gamma=W^{T} X,
$$

where $\quad W^{\mathrm{T}}=\left(\mathrm{X}_{1}, \mathrm{X}_{2}, \ldots, \mathrm{X}_{d}\right)^{T}=\left(P Y_{1}, P Y_{2}, \ldots, P Y_{d}\right)^{T}=\left(Y_{1}, Y_{2}, \ldots, Y_{d}\right)^{T} P^{T}$

The transformation in (4) can be divided into two items $Y=P^{T} \mathrm{X} \quad$ and $\Gamma=V^{T} Y \quad$, where $\quad V=\left(Y_{1}, Y_{2}, \ldots, Y_{d}\right)$, $\mathrm{P}=\left(\beta_{1}, \beta_{2}, \ldots, \beta_{m}\right) . \mathrm{V}$ is the eigen matrix by training, and each $G_{i}^{j}$ can transformed into a vector by (5) as follows:

$$
\begin{aligned}
& Y_{i}^{j}=P^{T} G_{i}^{j} \\
& F_{1}=V^{T} Y_{i}^{j}
\end{aligned},
$$

By using the same method, the eigen matrix of Region bounded by Legs $\left(V_{r b}\right)$ is obtained, each image of Region bounded by Legs ( $L_{i}^{j}$ ) corresponding $G_{i}^{j}$ can obtained $F_{2}$ by (6) as follows, and it can represent an image of legs region

$$
\begin{aligned}
& M_{i}^{j}=P^{T} L_{i}^{j} \\
& F_{2}=V_{r b l}^{T} M_{i}^{j},
\end{aligned}
$$

\section{Representation}

Since the gait of a person varies slightly due to change in carrying conditions, change in clothes and different normal conditions, and we noticed that the RBL has significant discriminative information, so the features of GEI and the features of RBL can combine into one kind of features to represent gait features. The method which is proposed above is used to extract features of GEI and images of RBL, and $G_{i}^{j}$ can be represent as follows:

$$
G_{i}^{j}=\left[\left\{F_{1}\right\},\left\{F_{2}\right\}\right],
$$

where $F_{1}$ and $F_{2}$ are the features of entire GEI and the features of images of RBL respectively.

$$
\begin{aligned}
& F_{1}=\left\{f_{i_{1}}^{j}, f_{i_{2}}^{j}, \ldots ., f_{i_{d}}^{j}\right\}, \\
& F_{2}=\left\{f_{i_{d+1}}^{j}, f_{i_{d+2}}^{j}, \ldots ., f_{i_{2 d}}^{j}\right\},
\end{aligned}
$$

The same method is also used to extract the features of testing samples. The features of testing samples can represent as follows:

$$
\begin{aligned}
& G_{i}=\left[\left\{P F_{1}\right\},\left\{P F_{2}\right\}\right], \\
& P F_{1}=\left\{t_{1}, t_{2}, \ldots, t_{d}\right\}, \\
& P F_{2}=\left\{t_{d+1}, t_{d+2}, \ldots, t_{2 d}\right\},
\end{aligned}
$$

where $P F_{1}$ is the features of entire GEI of testing samples, and $\mathrm{PF}_{2}$ is the features of images of RBL of test samples.

\section{Classification}

In order to recognize a test gait image $G_{i}$, the minimum distance classifier is used. The distance between testing image and training image are calculated, and if the distance between $G_{i}$ and $G_{i}^{j}$ is minimum, so $G_{i}$ and $G_{i}^{j}$ belong to the same class. Euclidean distance formula (17) is used to calculate the distance between testing samples and training samples.

$$
d\left(G_{i}, G_{i}^{j}\right)=\left[\sum_{n=1}^{2 d}\left(f_{i n}^{j}-t_{n}\right)^{2}\right]^{\frac{1}{2}},
$$

where $i=1, \cdots, C, j=1, \cdots, N_{i}$.

\section{EXPERIMENTAL RESULTS}

In order to verify the performance of the proposed method of gait recognition, we have conducted an experiment on the standard CASIA A, CASIA B and CASIA C.

Firstly, the original gait images in 90 degree viewing angle of CASIA A are selected to be removed noise, be detected body area, and be normalized. Then these images can be transformed into GEI by (1). The CASIA A has become a dataset which consists of 20 subjects, each subject has 18 GEI. The GEI which is transformed are used to train and test. 1/6, $1 / 3,1 / 2,2 / 3,5 / 6$ of GEI of samples are selected to train, respectively, the remaining are used to test, the optimal recognition rate is recorded by adjusting $\eta$ and $d$. Some experiment results show that when 2/3of GEI of samples are used to train, the best recognition is obtained.

In order to prove the significant discriminative information with RBL, we choose walking with bag condition, walking with coat condition and normal walking condition for CASIA A and CASIA B. The values of the parameters are $\eta=0.17$, $\eta=0.26, \eta=0.32$ and $d=49,50,50$ when person in the three different conditions, respectively using PCA+RLDA. While values of the parameters are $\eta=0.12, \eta=0.21, \eta=0.26$ and $d=47$, 47, 45 when person in the three different conditions respectively in PCA+RBL+RLDA. Some experimental results are shown in Fig. 5, where (a) and (b) are the results of experiment on CASIA A and CASIA B, respectively.
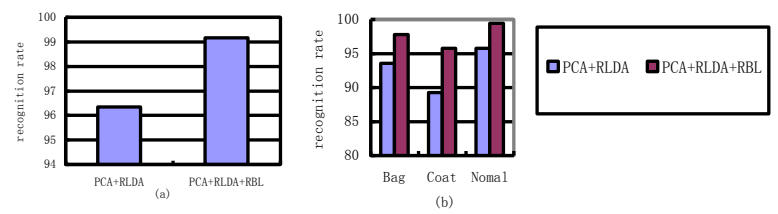

Fig. 3 The influence of RBL for recognition 
As we can see from Fig.3, recognition rate of PCA+RBL+RLDA is better than PCA +RLDA without RBL. So it is proved that he RBL has significant discriminative information.

In addition, the proposed method compared with the method based on Mutual Information ${ }^{[6]}$, the method based on $\mathrm{LBP}+\mathrm{RBL}^{[7]}$, and the method based on $\mathrm{PCA}+\mathrm{LDA}^{[11]}$. In this experiment, 2/3 of GEI of samples are used to train and the remaining is used to test. TABLE 1 shows the optimal recognition rate.

TABLE 1 The comparison of three different methods

\begin{tabular}{|c|c|c|c|}
\hline Method & $\begin{array}{l}\text { Recognition } \\
\text { rate }\end{array}$ & $\begin{array}{c}\text { Running } \\
\text { time }\end{array}$ & Dataset \\
\hline \multirow{5}{*}{$\begin{array}{l}\text { Method based } \\
\text { on Mutual } \\
\text { Information }\end{array}$} & $83.30 \%$ & 13.1 & CASIA A \\
\hline & & & CASIA B \\
\hline & $78.30 \%$ & 31.3 & Walking with bag \\
\hline & $82.70 \%$ & 37.9 & Walking with coat \\
\hline & $85.40 \%$ & 33.5 & Normal walking \\
\hline \multirow{5}{*}{$\mathrm{LBP}+\mathrm{RBL}$} & $100 \%$ & 20.7 & CASIA A \\
\hline & & & CASIA B \\
\hline & $95.82 \%$ & 45.2 & Walking with bag \\
\hline & $96.31 \%$ & 47.9 & Walking with coat \\
\hline & $100 \%$ & 44.3 & Normal walking \\
\hline \multirow{5}{*}{$\begin{array}{c}\mathrm{PCA}+\mathrm{LDA}+ \\
\text { RBL }\end{array}$} & $100 \%$ & 13.6 & CASIA A \\
\hline & & & CASIA B \\
\hline & $97.70 \%$ & 33.5 & Walking with bag \\
\hline & $95.13 \%$ & 35.2 & Walking with coat \\
\hline & $100 \%$ & 34.8 & Normal walking \\
\hline \multirow{5}{*}{$\begin{array}{c}\text { PCA+RLDA } \\
\quad+\text { RBL }\end{array}$} & $99.16 \%$ & 13.5 & CASIA A \\
\hline & & & CASIA B \\
\hline & $97.78 \%$ & 32.0 & Walking with bag \\
\hline & $95.77 \%$ & 34.6 & Walking with coat \\
\hline & $99.46 \%$ & 34.2 & Normal walking \\
\hline
\end{tabular}

TABLE 2 The recognition rate of some different methods on CASIA C

\begin{tabular}{|c|c|c|}
\hline Method & Recognition rate & Condition \\
\hline LBP+RBL & $93.27 \%$ & Normal walking \\
\hline \multirow{4}{*}{} & $86.10 \%$ & Fast walking \\
\cline { 2 - 3 } & $86.35 \%$ & Slow walking \\
\hline \multicolumn{2}{|c|}{ The average recognition rate } & Walking with bag \\
\hline PCA+LDA+RBL & $97.35 \%$ & Normal walking \\
\hline & $94.54 \%$ & Fast walking \\
\cline { 2 - 3 } & $95.50 \%$ & Slow walking \\
\cline { 2 - 3 } & $91.09 \%$ & Walking with bag \\
\hline \multicolumn{2}{|c|}{ The average recognition rate } & $94.64 \%$ \\
\hline PCA+RLDA+RBL & $98.01 \%$ & Normal walking \\
\hline \multicolumn{2}{|c|}{ The average recognition rate } & Fast walking \\
\cline { 2 - 3 } & $95.31 \%$ & Slow walking \\
\cline { 2 - 3 } & $95.44 \%$ & Walking with bag \\
\hline
\end{tabular}

As we can see from TABLE 1, the recognition rate of proposed method is better than the other methods, and the running time is less than $\mathrm{LBP}+\mathrm{RBL}$ and $\mathrm{PCA}+\mathrm{LDA}+\mathrm{RBL}$, and approximately with the method based on Mutual Information, however, the recognition rate is more better than it.

To verity the robustness of the proposed method, the CASIA $\mathrm{C}$ is used to experiment. Because of the images of it is shoot at night by infrared camera. So it has some different from the other two Datasets in brightness and contrast ratio, then the recognition rate will be affected. TABLE 2 shows the result.

\section{CONCLUSION}

In this paper, we propose a PCA+RBL+RLDA method for gait recognition. The features are extracted from entire GEI and the images of RBL, respectively. Then the two parts of features are combined to represent a gait sample. In order to verify the performance of the proposed method of gait recognition, we conduct some experiments on the standard CASIA A, CASIA B and CASIA C. Meanwhile, we compare with the other methods to demonstrate the effectiveness of the proposed method. In addition, some experiments on the standard CASIA $\mathrm{C}$ are conducted to show the robustness of proposed method.

\section{REFERENCES}

[1] Zheng S, Huang K Q, Tan T N, Tao D C.A cascade fusion scheme for gait and cumulative foot pressure image recognition. Pattern Recognition, 2012, 45(10):3603-3610

[2] Hu H F. Enhanced gabor feature based classification using a regularized locally tensor discriminant model for multiview gait recognition. IEEE Transactions on circuits and systems for video technology, 2013, 23(7):1274-1286

[3] Bashir K, Xiang T, Gong S G. Gait recognition without subject cooperation. Pattern Recognition Letter, 2010, 31(13):2052-2060

[4] Han J, Bhanu B. Individual recognition using gait energy image. IEEE Transactions on Pattern analysis and Machine Intelligence, 2006 28(2):316-322

[5] Roy A, Sural S, Mukherjee J. Gait recognition using pose kinematics and pose energy image. Signal Processing, 2012, 92(3):780-792

[6] Guo B F, M S Nixon. Gait feature subset selection by mutual information. IEEE Transactions on Systems, Man, and Cybernetics-Part A: Systems and Humans, 2009, 39(1):36-46

[7] Mohan Kumar H P, Nagendraswamy H S. LBP for gait recognition: a symbolic method based on GEI plus RBL of GEI. Electronics and Communication Systems (ICECS), 2014 International Conference on. IEEE, 2014: 1-5.

[8] Kusakunniran W, Wu Q, Zhang J, Li H D. Gait recognition under various viewing angles based on correlated motion regression. IEEE Transactions on Circuits and Systems for Video Technology, 2012, 22(6):966-980

[9] Lu J W, PLATANIOTIS K.N.,VENETSANOPOULOS A N. Regularization studies of linear discriminant analysis in small sample size scenarios with application to face recognition. Pattern Recognition Letters, 2005, 26(2):181-191

[10] Yang J, Yang J Y. Why can LDA be performed in PCA transformed space? Pattern Recognition, 2003, 36(2):563-566

[11] $\mathrm{Hu} \mathrm{H} \mathrm{F.} \mathrm{Multiview} \mathrm{gait} \mathrm{recognition} \mathrm{based} \mathrm{on} \mathrm{patch} \mathrm{distribution} \mathrm{features}$ and uncorrelated multilinear sparse local discriminant canonical correlation analysis. IEEE Transactions on Circutts and Systems for Video Technology, 2014, 24(4):617-630 